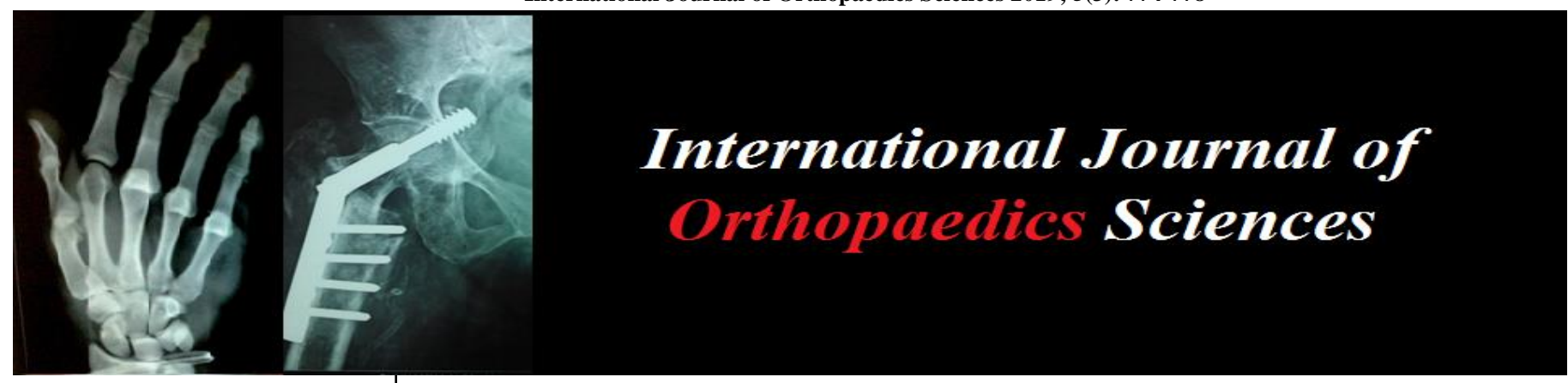

ISSN: $2395-1958$

IJOS 2019; 5(3): 774-778

(C) 2019 IJOS

www.orthopaper.com

Received: 24-05-2019

Accepted: 29-06-2019

Pranesh Kumar M

Senior Resident, Department of Orthopaedics, Kovai Medical

Centre and Hospital institute of health science and research,

Coimbatore, Tamil Nadu, India

Dr. Hari Sivanandan M

Assistant Professor, Department of Orthopaedics, Vinayaka

Missions Research Foundation,

Salem, Tamil Nadu, India

\section{Manoharan AE}

Professor, Department of Orthopaedics, Vinayaka Missions

Research Foundation, Salem,

Tamil Nadu, India

\section{Correspondence}

Dr. Hari Sivanandan M

Assistant Professor, Department of Orthopaedics, Vinayaka

Missions Research Foundation,

Salem, Tamil Nadu, India

\section{A prospective study of functional outcome of high tibial osteotomy}

\author{
Pranesh Kumar M, Dr. Hari Sivanandan M and Manoharan AE
}

DOI: https://doi.org/10.22271/ortho.2019.v5.i3m.1625

\section{Abstract}

Introduction: Osteoarthritis is a degenerative disease. It causes chronic disability; the degree of disability depends on the site involved. Knee Osteoarthritis is more common among all types of arthritic conditions. High tibial osteotomy is an accepted surgical technique for treatment of primary or secondary medial compartment degenerative arthritis of knee in younger and active patients. Hence this study is done to assess the functional outcome in patients undergoing High Tibial Osteotomy using knee society score and Functional score.

Methodology: This is a prospective interventional, single centre study was done on 60 patients who were clinically and radiologically diagnosed as Osteoarthritis with varus deformity. After proper preoperative assessment, patient underwent high tibial osteotomy. The clinical outcome was evaluated with knee society score and functional score. The patients were followed up at regular intervals during $3^{\text {rd }}, 6^{\text {th }}$ and at $12^{\text {th }}$ month.

Results: The preoperative mean knee score and the mean functional score of the patients before surgery were 54.3 and 53.22 respectively. The postoperative mean knee score and functional score at 12 months was 82.3 and 81.5 respectively. So the preoperative and postoperative comparison score showed statistically significant improvement in both the knee society score and the functional score.

Conclusion: In this study, there was significant increase in the knee score and functional score after high tibial osteotomy for the patients of osteoarthritis with Varus deformity. Therefore, High tibial osteotomy should be recommended for the treatment of degenerative arthritis of the knee in young, active patients for symptomatic improvement and maintenance of activity levels.

Keywords: Osteoarthritis, high tibial osteotomy, knee society score, functional score

\section{Introduction}

Osteoarthritis is a degenerative disease characterized by gradual development of joint pain, stiffness, swelling and limitation of movements. Osteoarthritis causes chronic disability; the degree of disability depends on the site involved and varies greatly between individual [1]. During early stages of osteoarthritis, non-surgical treatment options include weight reduction, low impact activity and physiotherapy. As the disease progress to end stage osteoarthritis, the surgical treatment options are high tibial osteotomy, unicompartmental arthroplasty and total knee arthroplasty. Arthroplasty is considered a good option for healthier patient, older than 60 years with good long term outcome reported. However concern remains regarding the longevity of the implants in younger patients ${ }^{[2]}$.

High tibial osteotomy is an accepted surgical technique for treatment of medial compartment arthrosis of knee in younger patients. This procedure stands ahead of closed wedge osteotomy because the Peroneal nerve is not in jeopardy and there is no disruption of proximal tibiofibular joint and lateral collateral ligaments worth achievement of more precise correction [3]. The biomechanical principle of high tibial osteotomy is to redistribute the weight bearing forces from the worn medial compartment across to the lateral compartment thereby relieving pain and slowing the disease progression.

The main indication for high tibial osteotomy is pain and angular deformity of the knee due to unicompartmental gonarthrosis. Most cases reported in the literature have medial gonarthrosis with varus deformity of the knee, but about 15 percent of the subjects have lateral gonarthrosis with valgus deformity. Two main treatments have been available: closed wedge osteotomy and dome (vault) osteotomy. 
Normally, the knee is overcorrected to 5-13 degrees of valgus in medial gonarthrosis, and the opposite in cases of lateral gonarthrosis. This overcorrection is important for the final result of the osteotomy ${ }^{[4,5]}$. Another possible mechanism may be reduction of the intraosseous pressure. Outcome is an evaluation of observations associated with a study period with respect to factors that can be of interest after some kind of intervention. The goals must be expressed in terms of reduction of disablement, and they must be measurable ${ }^{[6,7,8]}$. Whether a treatment is effective or not must be judged from the degree of goal achievement. Even if there is a statistically significant improvement, it is inadequate if the treatment goal is not reached.

In the present study, outcome after high tibial osteotomy has been evaluated with respect to postoperative improvement and individual goal achievement.

\section{Materials and Methods}

It is a prospective interventional single centre study conducted in Vinayaka Missions Kirupananda Variyar Medical College and Hospital, Salem, during the period between August 2013 to august 2015 after permission from Instituitional Ethical committee. The patients included were those who had standard indication for High tibial osteotomy. Criteria to include the patients in this series were, Unicompartmental Arthritis of knee with varus deformity, good muscle strength to carry out rehabilitation and good vascular status without arterial insufficiency. Exclusion Criteria were patients who lost follow-up, ligamentous instability, lateral tibial subluxation of more than $1 \mathrm{~cm}$, medial compartment tibial bone loss of $>2$ or $3 \mathrm{~mm}$, flexion contraction of $>15^{\circ}$, knee flexion $<90^{\circ},>20^{\circ}$ of correction needed and rheumatoid arthritis. About 60 patients were selected and were assessed preoperatively and postoperatively with Knee Society score and Functional Score sheet. Assessment and evaluation was done using a regularized custom made protocol which included the symptoms of the patients, associated medical conditions, knee society score and knee society functional score. The Knee Society Score is a special score which has been widely accepted as an objective measure of knee status in patients undergoing High Tibial Osteotomy. The Knee flexion is measured using Goniometer. All the data are entered into the protocol proforma. $\mathrm{X}$ rays included standing anteroposterior view and lateral view of the knee. Postoperative $X$ ray were done immediate postoperative and after 1 month, 6 months and yearly thereafter.

\subsection{Statistical analysis}

Data's are entered and analyzed by statistical analysis using SPSS version 16.Mean, Standard Deviation, Chi-square test are used to analyse the result and test the significance

\section{Results}

Table 1 shows that the age of the patients had ranged from 45 - 50 years with a mean age of 47.8 years. Among the 60 patients 37 were females and only 23 were males. For majority of the patients right side of the knee $(56.6 \%)$ was involved and for about 5 patients bilateral knee was involved. $70 \%$ of the patients had grade III type of Osteoarthritis and only $30 \%$ had grade IV type of Osteoarthritis (Table 2). The mean knee score was 54.3 and the mean functional score was 53.2 (Table 3). Both the mean scores was considered as poor based on the categorization of the scores. The immediate postoperative infection was the wound infection which had developed in around $5-7$ days. 5 patients had developed the infection out of 60 and for the remaining 55 patients the wound was clear without any infections. Infection is of serous discharge for all 5 patients. The patients who had infection were treated with high level antibiotics for 5 days and at the end of 1 week the discharge was cleared and wound was healthy. Out of 60 patients only one patient had developed the foot drop due to the common peroneal nerve injury. For that patient proper braces were given to improve the condition. Table 4 shows that all the patients were followed for a period of 1 year at the interval of 3, 6 and 12 months. Each visits the patients knee score and functional score was assessed using the standard orthopaedics knee society protocol. The grading of the knee society was done as score below 60 was considered as poor, score $60-69$ was considered as fair, score $70-79$ was good and score $80-100$ is excellent. It is inferred from the table that the patients total knee score was gradually improved over the period of 1 year. The score was initially in the range of fair to good and at the end of 1 year it was excellent $(>80)$. The improvement in the score over the period of 1 year was proven to be statistically significant $(p<.0001)$. The knee society score at the end of 3 months was 63.1 and at end 12 months was 82.3. The patient's functional score had gradually improved over the period of 1 year. The initial score at the end of 3 months was 62.9 and at the end of 12 months it was 81.5 and this improvement in the functional knee score over the period of 12 months was found to be statistically significant $(<.0001)$

Table 5 shows that the patients who were in the grade of poor based on the total knee score and functional score before the high tibial osteotomy had improved to excellent after the surgery.

Table 1: Age wise distribution of the study population

\begin{tabular}{|c|c|c|c|}
\hline Age in years & Frequency & Percentage & Mean Age (in years) \\
\hline 45 & 6 & $10 \%$ & \\
\hline 46 & 8 & $13.3 \%$ & \\
\hline 47 & 11 & $18.3 \%$ & \\
\hline 48 & 11 & $18.3 \%$ & \multirow{2}{*}{47.8} \\
\hline 49 & 14 & $23.3 \%$ & \\
\hline 50 & 10 & $16.6 \%$ & \\
\hline Total & 60 & $100 \%$ & \\
\hline
\end{tabular}

Table 2: Distribution of the study population based on grading of Osteoarthritis by Kellgren and Lawrence

\begin{tabular}{|c|c|c|}
\hline Grading of Osteoarthritis & Frequency & Percentage \\
\hline Grade III & 42 & $70 \%$ \\
\hline Grade IV & 18 & $30 \%$ \\
\hline Total & 60 & $100 \%$ \\
\hline
\end{tabular}

Table 3: Mean and Standard Deviation of the Knee society score and the functional score of the patients before surgery

\begin{tabular}{|c|c|c|}
\hline Score & Mean & Standard Deviation \\
\hline Knee score & 54.3 & 2.15 \\
\hline Functional score & 53.2 & 2.37 \\
\hline
\end{tabular}

Table 4: Mean and Standard Deviation of the knee society score and functional score among the study population after high tibial osteotomy

\begin{tabular}{|c|c|c|c|c|}
\hline \multirow[b]{2}{*}{ Score } & \multicolumn{3}{|c|}{ Postoperative period (Mean \pm SD) } & \multirow[t]{2}{*}{ P Value } \\
\hline & 3 months & 6 months & 12 months & \\
\hline Knee Society Score & $63.1 \pm 3.9$ & $72.5 \pm 5.8$ & $82.3 \pm 6.2$ & $<.0001$ \\
\hline Functional score & $62.9 \pm 3.9$ & $72.2 \pm 5.6$ & $81.5 \pm 6.2$ & $<.0001$ \\
\hline
\end{tabular}


Table 5: Mean and Standard Deviation of the Knee society Score and Functional score before and after high tibial osteotomy.

\begin{tabular}{|c|c|c|c|}
\hline Score $($ Mean \pm SD) & Before surgery & $\begin{array}{c}\text { After surgery } \\
\text { (end of 12 months) }\end{array}$ & $\begin{array}{c}\text { P } \\
\text { value }\end{array}$ \\
\hline Knee Society Score & $54.3 \pm 2.15$ & $82.3 \pm 6.2$ & $<.0001$ \\
\hline Functional score & $53.2 \pm 2.37$ & $81.5 \pm 6.2$ & $<.0001$ \\
\hline
\end{tabular}

$\mathrm{P}$ value derived by applying paired $\mathrm{T}$ test

\section{Discussion}

The present study had shown a favorable outcome for high tibial osteotomy. The 60 patients who had undergone lateral closing wedge high tibial osteotomy for osteoarthritis with Varus deformity had shown a statistically significant improvement in both the knee score and the functional score at the end of 1 year follow-up following the surgery. Out of 60 patients only one patient had developed a foot drop due to common peroneal nerve injury, as a post-operative complication. Whereas other than that for the remaining 59 patients the entire post-operative period was uneventful.

Aglietti et al. [9] performed 139 osteotomies with three different techniques: (1) lateral closed wedge osteotomy, without internal fixation followed by long leg cast; (2) lateral closed wedge or "en chevron" osteotomy, fixed with two screws and immobilized with a long leg cast; and (3) Lateral closed wedge osteotomy according to Insall et al. ${ }^{[10]}$ without internal fixation and with cylinder cast immobilization. The outcomes were satisfactory in $87 \%$ (from 2 to 5 years of follow-up), in $70 \%$ (from 6 to 10 years of follow-up) and in $64 \%$ (more than 10 years of follow-up). The authors concluded that the third group of patients had better results and correction, that severe articular destruction had poorer outcomes and that under corrected knees tended to relapse.

Matthews et al. ${ }^{[11]}$ treated 40 patients with Coventry or Coventry-Bowman techniques followed by cast immobilization. Internal fixation with staples was used in 25 cases and external fixation in three cases. They reported $86 \%$ of satisfactorily results at one year from surgery, $64 \%$ at three years, $50 \%$ at five years and $28 \%$ at nine years. They also concluded that obesity, advanced age, overcorrection or under correction had the worst outcomes.

Ivarsson et al. ${ }^{[12]}$ performed 99 lateral closing wedge High Tibial Osteotomy, fixed with staples and immobilized in a cast. They reported $75 \%$ of good and acceptable outcomes at 5.7 years and $60 \%$ at 11.9 years. They obtained better results in patients with Ahlbäck grade I or II osteoarthritis and when a slight overcorrection was achieved (from $3^{\circ}$ to $7^{\circ}$ of valgus). Naudie et al. ${ }^{[13]}$ performed 94 closing wedge osteotomies and 12 dome osteotomies, fixed with staples and followed by cast immobilization in 75 cases. The Kaplan-Meier analysis showed survivorship of $75 \%$ at five years, $51 \%$ at ten years, $39 \%$ at 15 years and $30 \%$ at 20 years. However, in patients younger than 50 years old and with range of motion $>120^{\circ}$, the longevity increased to $80 \%$ at ten years. They thus underlined the importance of correct patient selection. In their series, earlier failure was associated with age $>50$ years, previous arthroscopic debridements, lateral tibial thrust, preoperative knee flexion $<120^{\circ}$, under correction and delayed union.

Sprenger and Doerzbacher ${ }^{[14]}$ treated 76 knees with closing wedge High Tibial Osteotomy and internal fixation. Survival rates were $65-74 \%$ at ten years from surgery. However, tenyear survivorship was $90 \%$, when the femurotibial angle was between 8 and $16^{\circ}$ valgus at one year from surgery.

Koshino et al. ${ }^{[10]}$ performed 75 closing wedge High Tibial Osteotomy, fixed by external fixation, internal fixation or long leg cast. The survivorship reported was $97.3 \%$ at seven years, $95.1 \%$ at ten years and $86.9 \%$ at 15 years from surgery.

Tang and Henderson ${ }^{[15]}$ treated 67 knees with lateral closing High Tibial Osteotomy, fixed with staples or plate or immobilized in a long leg cast. Survival rates reported were: $89.5 \%$ at five years, $74.7 \%$ at ten years and $66.9 \%$ for 15 and 20 years.

Asik et al. ${ }^{[16]}$ performed 65 open wedge osteotomies fixed with the Puddu plate. They reported significant improvement of pain and knee function at an average follow-up of 34 months.

Chiang et al. ${ }^{[17]}$ used dome-shaped High Tibial Osteotomy and external fixation to treat 25 knees with medial compartment arthrosis. In their series, the Hospital for Special Surgery (HSS) score was excellent or good in 18 knees at five years and in 13 knees at an average of 15 years.

Papachristou et al. ${ }^{[18]}$ followed up 44 closing wedge High Tibial Osteotomy, fixed with one or two staples. Survivorship analysis showed a success rate of $80 \%$ at ten years, $66 \%$ at 15 years and $53 \%$ at 17 years from surgery.

Flecher et al. ${ }^{[19]}$ followed up 301 patients treated with closing wedge High Tibial Osteotomy and internal fixation. Survivorship was $85 \%$ at 20 years. They also concluded that the most important risk factors predicting revision (the chosen endpoint) were: age $>50$ years and preoperative Ahlbäck grade III or more arthrosis.

Gstöttner et al. ${ }^{[20]}$ treated 134 arthritic knees with lateral closing High Tibial Osteotomy, fixed with staples. The survival rates were $94 \%$ at five years, $79.9 \%$ at ten years, $65.5 \%$ at 15 years and $54.1 \%$ at 18 years.

Akizuki et al. ${ }^{[21]}$ followed up 118 closing wedge High Tibial Osteotomy, fixed with a plate. Survivorship was $97.6 \%$ at ten years and $90.4 \%$ at 15 years from surgery.

The results of valgus tibial osteotomy for knee osteoarthritis have been extensively reported in the literature. A medial compartment osteoarthritis in a young patient with good arc of motion is expected to give a satisfactory motion, better function with less pain in $80 \%$ of the time after five years. This would, however, deteriorate with time ${ }^{[22]}$. Arthroplasty, however, despite excellent pain relief and improved function, would not be a life-long remedy for a young person ${ }^{[23]}$. In addition, it is expensive and also imposes some functional limitations-things which are not always acceptable in developing countries. Open medial wedge osteotomies preserve better bone stock, and cause less patella infra, thus providing an easier future conversion to knee arthroplasty ${ }^{[24]}$. This kind of osteotomy is, however, a more extensive surgery, with more possible complications; and the long-term results are not clearly evident yet.24 Unicompartmental replacement for medial osteoarthritis has had its share of enthusiasm and rejection over the years ${ }^{[25]}$. The difficulty in revising a unicompartmental to a total arthroplasty, has been the concern for some people, making them favor High Tibial Osteotomy over unicompartmental replacement for medial compartment osteoarthritis. The newer designs of uni-compartment replacement seem to take out much less bone, and the results, when used with proper indication, are encouraging ${ }^{[13]}$. The economic issues and yet-unclear long-term results are some of the problems with unicompartment arthroplasty. The more common patterns of reporting the end results of knee surgery procedures in the past were on the basis of radiographic changes, range of motion of knee or a rough patient's subjective satisfaction ${ }^{[26]}$. It has now become clear that pure objective data in many disease conditions and in most 
musculoskeletal illnesses are not reliable reflections of patient's improvement or regression. The way a patient functions and the change that is produced in his/her functions following an intervention seem to be better parameters to evaluate. For this reason, many scales of assessments have been designed to measure the patients' general feeling about their own health and their feelings regarding a particular disease, such as knee osteoarthritis, and also the achievement of their expectations after an intervention (such as tibial osteotomy or knee replacement for osteoarthritis of knee) ${ }^{[27]}$. The degree of valgus alignment obtained at surgery and, more so, the remaining valgus alignment with passage of time has been reported as positive factors affecting the results. Almost $90 \%$ of our patients had maintained their valgus alignment.

High Tibial Osteotomy in social settings, where floor sitting (rather than chair sitting) and use of toilets without seat are common, and also where economy, in terms of healthinsurance courage, are important issues, is one of the useful armamentarium in the hand of an orthopedic surgeon. An improvement in functional scoring, particularly in items such as pain, surface walking, use of Persian-style toilets, floor sitting, shopping, participation in social activities and selfesteem are to be expected in over the years period.

\section{Conclusion}

The main improvements seen in this study were related to pain reduction and an increase in the knee score and functional score after high tibial osteotomy and also delays the need for total knee replacement. Hence it is indisputable that appropriate patient selection, precise surgical planning and various operative techniques provide a favourable outcome of High Tibial Osteotomy in medial knee arthritis in young or middle-aged active patients.

\section{Conflict of interest: None}

\section{Acknowledgement: None}

\section{References}

1. Shah SN, Anand MP, Acharya VN, Karnad DR, Bichile SK, Kamath SA et al. API Text book of Medicine. Vol.2. 9th ed. Mumbai: The Association of Physicians of India, 2012, 1818.

2. Catherine Hui, Lucy Salmon J, Alison Kok, Heidi Williams A, Niels Hockers, Willem M. van der Tempel, Rishi Chana, Leo A. Pinczewski. Long-Term Survival of High Tibial Osteotomy for Medial Compartment Osteoarthritis of the Knee. The American Journal of Sports Medicine. 2011; 39(1):64-70.

3. Su Chan Lee, Kwang Am Jung, Chang Hyun Nam, Soong Hyun Jung, Seung Hyun Hwang. The Short-term Follow-up Results of Open Wedge High Tibial Osteotomy with Using an Aescula Open Wedge Plate and an Allogenic Bone Graft: The Minimum 1-Year Followup Results. Clinics in Orthopedic Surgery. 2010; 2:47-54.

4. Keene JS, Dyreby Jr JR. High tibial osteotomy in the treatment of osteoarthritis of the knee. The role of preoperative arthroscopy. J Bone Joint Surg Am. 1983; 65:36-42.

5. Keene JS, Monson DK, Roberts JM, Dyreby Jr JR. Evaluation of patients for high tibial osteotomy. Clin Orthop, 1989, 157-65.

6. Echternach JL, Rothstein JM. Hypothesis-oriented algorithms. Phys Ther. 1989; 69:559-64.

7. Bennecom CAM, Jelles F, Lankhorst GJ. Rehabilitation activity profilelMa brief research report on an application of the ICIDH in rehabilitation medicine. Eur. J Phys. Med Rehabil. 1993; 3:125-6.

8. Craik RL. Disability following hip fracture. Phys Ther. 1994; 74:387-98.

9. Aglietti P, Rinonapoli E, Stringa G, Taviani A. Tibial osteotomy for the varus osteoarthritic knee. Clin Orthop Relat Res. 1983; 176:239-251.

10. Bauer GC, Insall J, Koshino T. Tibial osteotomy in Gonoar-Throsis (Osteo-arthritis of the knee). J Bone Joint Surg Am. 1969; 51:1545-63.

11. Matthews LS, Goldstein SA, Malvitz TA, Katz BP, Kaufer H. Proximal tibial osteotomy. Factors that influence the duration of satisfactory function. Clin Orthop Relat Res. 1988; 229:193-200.

12. Ivarsson I, Myrnerts R, Gillquist J. High tibial osteotomy for medial osteoarthritis of the knee. A 5 to 7 and 11 year follow-up. J Bone Joint Surg Br. 1990; 72:238-244.

13. Naudie D, Boume RB, Rorabeck $\mathrm{CH}$, Bourne TJ. The install award. Survivorship of the high tibial valgus osteotomy. A 10 - 22 year follows up study. Clin Orthop Relat Res, 1999.

14. Sprenger TR, Doerzbacher JF. Tibial osteotomy for the treatment of varus gonarthrosis. Survival and failure analysis to twenty-two years. J Bone Joint Surg Am. 2003; 85-A:469-474.

15. Tang WC, Henderson IJP. High tibial osteotomy: long term survival analysis and patients' perspective. Knee. 2005; 12:410-413. DOI: 10.1016/j.knee.2005.03.006.

16. Asik M, Sen C, Kilic B, Goksan SB, Ciftci F, Taser OF. High tibial osteotomy with Puddu plate for the treatment of varus gonarthrosis. Knee Surg Sports Traumatol Arthrosc. 2006; 14:948-954. DOI: 10.1007/s00167-0060074-1.

17. Chiang H, Hsu H, Jiang C. Dome-shaped high tibial osteotomy: a long-term follow-up study. J Formos Med Assoc. 2006; 105(3):214-219. DOI: 10.1016/S09296646(09)60308-9.

18. Papachristou G, Plessas S, Sourlas J, Levidiotis C, Chronopoulos E, Papachristou C. Deterioration of longterm results following high tibial osteotomy in patients under 60 years of age. Int. Orthop. 2006; 30:403-408. DOI: 10.1007/s00264-006-0098-7.

19. Flecher X, Parratte S, Aubaniac JM, Argenson JN. A 1228-year followup study of closing wedge high tibial osteotomy. Clin Orthop Relat Res. 2006; 452:91-96. DOI: 10.1097/01.blo.0000229362.12244.f6.

20. Gstöttner M, Pedross F, Liebensteiner M, Bach C. Longterm outcome after high tibial osteotomy. Arch Orthop Trauma Surg. 2008; 128(1):111-115.

21. Akizuki S, Shibakawa A, Takizawa T, Yamazaki I, Horiuchi $\mathrm{H}$. The long-term outcome of high tibial osteotomy: a ten- to 20-year follow-up. J Bone Joint Surg Br. 2008; 90(5):592-596. DOI: 10.1302/0301620X.90B5.20386.

22. Meding JB, Kearing EM, Ritter MA et al. Total knee arthroplasty after high tibial osteotomy. Clin orthop. 2000; (375):175-84.

23. Wright RJ, Sledge CB, Poss $\mathrm{R}$ et al. Patient-reported outcome and survivorship after Kinemax total knee arthroplasty. J Bone Joint Surg Am. 2004; 86-A:2464-70.

24. Patond KR, Lokhande AV. Medial open wedge high tibial osteotomy in medial compartment osteoarthrosis of the knee. Natl Med J India. 1993; 6:104-8.

25. Rajasekhar C, Das S, Smith A. Unicompartmental knee arthroplasty. 2-to 12-year results in a community 
hospital. J Bone Joint Surg Br. 2004; 86:983-5.

26. Choi HR, Hasegawa $\mathrm{Y}$, Kondo S, et al. High tibial osteotomy for varus GonoarThrosis: A 10-to 24-year follow-up study. J Orthop Sci. 2001; 6:493-7.

27. Tugwell P, Bombardier C, Buchanan WWet al. The MACTAR Patient Preference Disability Questionnairean individualized functional priority approach for assessing improvement in physical disability in clinical trials in rheumatoid arthritis. J Rheumatol. 1987; 14:44651. 